\title{
Cuantificación de la carga de entrenamiento y competición: análisis comparativo por posiciones en un equipo de la Liga Española de Baloncesto Oro Quantification of training and competition load: comparative analysis by position in a Spanish Golden Basketball League team
}

*José Antonio Blázquez López, **Salvador García Martínez, **Alberto Ferriz Valero, *Javier Olaya Cuartero * Universidad Isabel I (España), ** Universidad de Alicante (España)

Resumen. El objetivo principal de este trabajo es cuantificar y analizar la carga de entrenamiento y competición de los jugadores de un equipo profesional de baloncesto de la categoría LEB Oro. Durante el estudio se han llevado a cabo procesos de análisis de carga objetiva y subjetiva mediante cuestionarios diarios de escalas de esfuerzo percibido (RPE) y control de minutos totales de entrenamiento y competición, obteniendo variables de carga media, semanal y total, índices de monotonía y fatiga y ratio de carga aguda-crónica. Se cuantificó la carga de entrenamiento y competición de 10 jugadores de un mismo equipo profesional $(\mathrm{edad}=27.2 \pm 5.3$ años; altura $=196.4 \pm 8.7 \mathrm{~cm}$; peso $=91.1 \pm 12.3 \mathrm{~kg})$ diferenciando entre bases, exteriores (escoltas y aleros) e interiores (ala-pívots y pívots) durante 21 semanas de entrenamiento. Los resultados principales del presente estudio mostraron mayor percepción de la carga para la posición de los bases en comparación con los interiores y exteriores. La mayoría de los jugadores de la plantilla se mantuvieron durante toda la primera vuelta en un estado de forma óptimo o sweet point, lo que supone una disminución del estado de fatiga y riesgo de lesión, debido a este seguimiento y control de la carga. Este estudio servirá a entrenadores y preparadores físicos a la hora de elegir diferentes herramientas para realizar una correcta cuantificación e interpretación de la carga de entrenamiento en un deporte de equipo como el baloncesto.

Palabras clave: deportes de equipo, jugadores profesionales, individualización, bajo coste, RPE.

\begin{abstract}
The main purpose of this study was to quantify and analyze the training and competition load of the players of a professional basketball team in «LEB Oro» category. During the study, objective and subjective load analysis processes have been carried out through daily Rating of Perceived Exertion (RPE) questionnaires and control of total training and competition minutes, getting variables of average, weekly and total load, training monotony and training fatigue indexes and acute: chronic workload ratio. The training and competition load of 10 players from the same professional team (age $=27.2 \pm 5.3$ years; height $=196.4 \pm 8.7 \mathrm{~cm}$; weight $=91.1 \pm 12.3 \mathrm{~kg}$ ) was quantified by differentiating point guards, perimeter players (shooting guards and forwards) and inside players (center and power forward) during 21 weeks of training. The main results of the present study showed a greater perception of the load for the position of the point guards in comparison with the inside players and perimeter players. Most of the players in the squad were kept in an optimal or sweet point state throughout the first part of the season, which means a decrease in the fatigue and risk of injury due to this monitoring and control of the load. This study will be useful for coaches and physical trainers when choosing different tools to carry out a correct quantification and interpretation of the training load in a team sport such as basketball.
\end{abstract}

Key words: team sports, professional players, individualization, low cost, RPE.

\section{Introducción}

En los deportes colectivos como el baloncesto la cuantificación de la carga de entrenamiento es una de las mayores preocupaciones de los entrenadores y preparadores físicos encargados del estado de forma de sus jugadores (Reina et al., 2019). Realizar este proceso supondrá una serie de beneficios muy notables que no se obtendrían si no se llevase a cabo dicho control y análisis (González-Badillo \& Ribas, 2002). El principal problema que se observa es la diferente adaptación de

Fecha recepción: 19-01-21. Fecha de aceptación: 06-06-21

Javier Olaya Cuartero

javier.olaya@ui1.es los deportistas ante los estímulos de trabajo, por presentar diferentes niveles de condición física, lo cual puede dar lugar a la aparición de fatiga, disminución del rendimiento deportivo o lesiones (Alexiou \& Coutts, 2008). Por lo tanto, la supervisión y cuantificación de la carga serán de gran importancia, puesto que es esencial para aspectos como determinar si los jugadores del equipo se están adaptando de forma adecuada al programa de entrenamiento, comprender las respuestas individuales y específicas que supone el entrenamiento, evaluar la fatiga y la necesidad de recuperación y descanso y minimizar el riesgo de lesiones y enfermedades (Halson, 2014).

Los deportes acíclicos basan sus acciones en patrones de movimiento muy específicos, que requieren ha- 
bilidades concretas tales como cambios de dirección o la capacidad de repetir aceleraciones entre otras, las cuales requieren el desarrollo de métodos de entrenamientos para desarrollar estas cualidades (Bishop et al., 2002). Concretamente, el baloncesto es considerado un deporte de carga intermitente, donde se intercalan acciones de baja, media y alta intensidad, produciéndose un gran número de cambios de dirección, saltos, distintos desplazamientos y sprints lineales y cortos, acciones todas ellas realizadas con o sin balón durante entrenamientos y partidos (Conte et al., 2015).

Evaluar diariamente las sesiones de entrenamiento, y con ello ajustar adecuadamente la carga, podría garantizar la consecución de los objetivos marcados por el cuerpo técnico (Vargas et al., 2015). Sin embargo, queda mucho por descubrir e investigar en el ámbito de la cuantificación de la carga a pesar de que pueda estar en auge, debido a que gran parte de la información obtenida sobre el seguimiento de los deportistas sigue procediendo de información anecdótica, de experiencias personales o directamente no está publicada (Bourdon et al., 2017). Por ejemplo, en este estudio se utilizan métodos sencillos y de bajo costo, algunos de ellos no conllevan ningún coste económico y son de fácil acceso e interpretación, además de no ser métodos invasivos, lo que facilitará todo el proceso de investigación. Para profundizar en el estudio se llevó a cabo la diferenciación de tres posiciones: bases, interiores y exteriores. Autores como Abdelkrim et al. (2007) destacan que conocer la posición que el jugador ocupa en la pista es algo fundamental para controlar la intensidad del entrenamiento porque puede haber posiciones donde el jugador realice esfuerzos más intensos que el resto de los jugadores, por ejemplo, el base. Por estas razones será necesario cuantificar la carga de entrenamiento.

González-Badillo y Ribas (2002) definen la carga de entrenamiento como el conjunto de exigencias psicológicas y biológicas provocadas por las actividades del entrenamiento realizado. Además, atendiendo a la clasificación de Navarro (1999) se pueden diferenciar la naturaleza, componentes, magnitud, organización, orientación y efectos como características de la carga.

Esta cuantificación de la carga puede realizarse de una manera subjetiva u objetiva. Respecto a la carga subjetiva, en diferentes estudios sobre cuantificación de la carga de entrenamiento se ha cotejado la utilidad de estas escalas de percepción del esfuerzo o Rating of Perceived Extertion (RPE) en relación con variables fisiológicas objetivas (Foster et al., 2001). En contraste, en los métodos objetivos no influye la percepción del deportista. Por ejemplo, la carga externa es una medida objetiva de trabajo que completa el deportista en cada competición o entrenamiento y que puede ser expresada en volumen (por ejemplo: kilómetros, unidad de tiempo o repeticiones) pero que es independiente de la carga interna (Mujika, 2012). Estudios que han llevado a cabo una comparación de métodos de la cuantificación de la carga en baloncesto, han encontrado correlación directa entre la carga obtenida mediante la observación subjetiva de las tareas de entrenamiento con los datos objetivos que proporcionan los sistemas objetivos de cuantificación de carga interna y externa (Reina et al., 2019). Durante las sesiones de entrenamiento los jugadores deben experimentar demandas similares a las de competición, por lo que los entrenadores deben conocerlas (Conte et al., 2015), aunque también cabe destacar que Montgomery et al. (2010) afirman que existen diferencias significativas entre el entrenamiento y la competición real en baloncesto. Por lo tanto, se requieren investigaciones que permitan obtener una serie de datos que ayuden, de manera práctica, a la hora de la toma de decisiones en cuanto a la planificación y adaptación del entrenamiento.

El objetivo general del presente estudio fue analizar la carga de entrenamiento y competición medida conjuntamente en un equipo de baloncesto profesional categoría LEB Oro (Liga Española de Baloncesto) durante 21 semanas de entrenamiento. Además, el estudio presentó otros objetivos específicos como diferenciar la carga de entrenamiento por posiciones (bases, interiores y exteriores), comprobar si los jugadores se mantuvieron en una ratio óptima de carga aguda y crónica durante la temporada y estudiar las diferentes herramientas para cuantificar la carga objetiva y subjetiva.

\section{Metodología}

\section{Muestra}

Participaron en este estudio voluntariamente $10 \mathrm{ju}$ gadores de baloncesto de un mismo equipo $($ edad $=$ $27.2 \pm 5.3$ años; altura $=196.4 \pm 8.7 \mathrm{~cm}$; peso $=91.1$ $\pm 12.3 \mathrm{~kg}$ ) de la categoría profesional española LEB Oro de distintas nacionalidades como española, estadounidense, letona, lituana y finlandesa. Los jugadores contaban con una media de 2.4 años de antigüedad en el club. Este equipo entrenaba cinco días a la semana y disputaba un partido oficial los fines de semana. Los criterios de inclusión para la participación del presente estudio fueron: pertenecer al equipo seleccionado durante todo el proceso de estudio, no estar lesionado más 
de un 50\% de las semanas del estudio y enviar una nota con la percepción subjetiva de esfuerzo de cada sesión mediante un formulario de Google Drive en una escala del 1 al 10. Tanto jugadores como cuerpo técnico fueron informados sobre todos los procedimientos de la investigación tales como: riesgos, beneficios, requisitos o desarrollo de este. De cada uno de ellos se obtuvo un escrito de consentimiento firmado con toda la información necesaria sobre el estudio en cuestión. El procedimiento fue aprobado por el comité de ética de la Universidad Isabel I de Castilla de Burgos.

\section{Procedimiento}

Para realizar el control de la carga de entrenamiento se llevó a cabo una recopilación de datos diariamente donde se recogían todas las variables (índice de monotonía y fatiga, carga media, semanal y total y ratio de carga aguda-crónica). Todos los datos obtenidos por parte de los sujetos seleccionados fueron registrados en una hoja de cálculo Excel ${ }^{\circledR}$ versión 1.0 de Windows con el fin de facilitar todo el proceso de recopilación de datos, además, este sistema de recogida permitió realizar operaciones de las distintas variables como el índice de fatiga o monotonía entre otros de manera muy sencilla. Además, de esta manera todos los datos obtenidos y reflejados en este documento pueden ser analizados fácilmente debido a que muestran un feedback inmediato. Respecto a la temporalización de este estudio, cabe destacar que, del total de las 21 semanas analizadas correspondientes a la primera vuelta de competición, las cinco primeras pertenecían a la etapa de pretemporada donde no había partidos de competición oficial, mientras que las 16 semanas siguientes pertenecían a la competición oficial acorde con las fechas oficiales de la Federación Española de Baloncesto donde se disputaba un partido cada fin de semana.

\section{Escala de Esfuerzo Percibido (RPE)}

La carga interna se cuantificó mediante la percepción subjetiva del esfuerzo. Se realizó al final de cada entrenamiento, de forma que cada atleta proporcionó una calificación de 1-10 de cómo les había parecido la intensidad de éste, siendo 1 el esfuerzo más bajo, y 10 el máximo (Foster, 1998; Gabbett, 2016). Cabe destacar que Foster (1998) diferencia entre la RPE y la RPE sesión, la primera mide el esfuerzo de una actividad o ejercicio concreto, mientras que la segunda mide el trabajo de la sesión completa de manera global. La valoración del esfuerzo global debe realizarse 30 minutos después de haber terminado la sesión según recomiendan
Uchida et al. (2014) debido a que, si se hace antes, los jugadores tienden a valorar la sesión teniendo en cuenta sobre todo la última tarea del entrenamiento, a causa de que se trata de la sensación más reciente. Este aspecto podría modificar la fiabilidad de los datos obtenidos por la variación de las notas recibidas por los jugadores. Siguiendo estas indicaciones se informó a los jugadores sobre la importancia de realizar la valoración en el momento más adecuado.

Para obtener la carga de entrenamiento de cada sesión se multiplicó la intensidad por la duración de este expresado en «unidades RPE $\times$ minutos» (Gabbett, 2016). De manera que se llevó a cabo un control de la carga interna de los jugadores, proporcionándoles a todos ellos un enlace web de Google Docs previamente elaborado al que podían acceder fácilmente mediante sus teléfonos móviles una vez finalizada la sesión de entrenamiento.

\section{Carga aguda y carga crónica}

Como refleja Gabbett (2016), la carga de entrenamiento aguda representa la fatiga y equivale a la última semana de entrenamiento (semana en la cual el equipo se encuentra en ese momento), mientras que la carga de entrenamiento crónica se corresponde al fitness y representa el promedio de las últimas 3-6 semanas de entrenamiento. Concretamente, en este estudio, estas variables se han calculado mediante un modelo denominado exponentially weighted moving averages (EWMA) que le da más importancia y pone más énfasis en las últimas sesiones realizadas (Williams et al., 2017) y que se ha demostrado que tiene mayor sensibilidad para detectar aumentos en la probabilidad de lesionarse que el modelo tradicional (Murray et al., 2017). Por tanto, para este cálculo, se ha seleccionado la última semana para obtener la carga aguda (7 días) y las últimas 4 semanas para obtener la carga crónica (28 días). Por último, para conocer la ratio de carga aguda-crónica se debe calcular primero el valor de EWMA para la carga aguda y para la carga crónica, y una vez se calculan estos valores, se divide EWMA aguda entre EWMA crónica. Cuando la carga de entrenamiento aguda es baja y la carga de entrenamiento crónica es alta estamos ante un buen estado de forma, sin embargo, cuando se trata del caso contrario, el deportista se encontrará en un estado de fatiga (Hulin et al., 2014). Lo importante de esta relación es que considera la carga de entrenamiento que el deportista ha realizado en lo que respecta a la carga para la que ha sido preparado, y calcular la ratio de carga aguda-crónica permitirá controlar el grado de 
fatiga y, por lo tanto, la probabilidad de aparición de lesiones. Los resultados de esta ratio permiten conocer si el deportista se encontraba en un estado de forma óptimo, que es aquel que se encuentra entre 0.7 y 1.3 , mientras que si estuviese por encima de 1.5 estaríamos ante un estado de fatiga, lo que supondría también, un mayor riesgo de lesión. Por lo tanto, los valores por debajo de 0.7 y por encima de 1.3 muestran que los jugadores no se encuentran en un estado de forma adecuado y deseado, excepto cuando estos cambios bruscos se realizan a propósito como parte de la planificación de entrenamiento (Gabbett, 2016).

\section{Índice de monotonía}

Una vez obtenida la carga de entrenamiento a través de la percepción subjetiva de esfuerzo se podrá obtener el índice de monotonía, el cual indica la variabilidad diaria del entrenamiento, que está relacionada con los síntomas del síndrome de sobreentrenamiento cuando son combinadas altas cargas de entrenamiento con un alto índice de monotonía (Foster, 1998). Se obtuvo mediante su fórmula, que consiste en dividir el promedio de las cargas de entrenamiento de la semana entre la desviación estándar. Cuanto más bajo sea el valor de desviación estándar, es decir, el valor de variabilidad, más cercano a 1 se encontrará el valor de índice de monotonía. Obtener valores de índice de monotonía de 1 o cercanos es algo positivo para el control de la progresión del entrenamiento, debido a que grandes variaciones mostradas mediante valores muy bajos o altos de índice de monotonía pueden suponer altos riesgos de lesión debido a que los cambios bruscos de las cargas de entrenamiento para los jugadores pueden ser perjudiciales (Foster, 1998).

\section{Índice de fatiga}

El índice de fatiga se obtuvo multiplicando la carga total por el índice de monotonía. Obtener esta variable permite detectar periodos con altos niveles de carga, lo que puede provocar un alto nivel de fatiga en los jugadores, por ello, cuanto mayor resulte ser, mayor riesgo de sobreentrenamiento tendrá el equipo (Foster, 1998). Mediante este seguimiento y control de la carga de entrenamiento se podrá reducir el riesgo de lesión, reduciendo las probabilidades de que los jugadores padezcan síntomas del síndrome de sobreentrenamiento.

\section{Análisis estadístico}

Todos los resultados son presentados como media (M) \pm desviación estándar (DE). Todas las variables cuan- titativas (carga total, índice de monotonía, carga media e índice de fatiga) fueron analizadas entre grupos (bases, exteriores e interiores) mediante un análisis de medidas independientes de la varianza (ANOVA) con un análisis posterior de Bonferroni. Esta prueba paramétrica se realizó tras comprobar la distribución normal de los datos mediante el test de Shapiro-Wilk y de no encontrar diferencias significativas en los tests de Levene para ninguna de las variables. La significación estadística fue establecida para $p<.05$. Además, se analizaron los tamaños del efecto debido al escaso tamaño muestral. Las magnitudes del tamaño del efecto fueron interpretadas mediante el criterio de Rhea (2004) atendiendo a la siguiente clasificación para sujetos entrenados profesionalmente: $<0.25$ trivial, $0.25-0.50$ pequeña, 0.50-1 moderada y $>1$ grande. Los datos fueron analizados usando el paquete estadístico SPSS para ciencias sociales (versión 24.0 para Windows, SPSS Inc, Chicago, IL, USA).

\section{Resultados}

En la Tabla 1 se pueden observar los valores de carga analizados por jugador y posición durante las 21 semanas de competición expresados como Media (M) \pm Desviación Estándar (DE). Exceptuando el «Base 1» todos los jugadores mostraron valores de carga media e índice de monotonía muy similares entre ellos. Respecto a la carga total, nuevamente todos mostraron valores muy parecidos exceptuando en este caso el jugador «Interior 1». El índice de fatiga fue el valor de mayor variación entre los jugadores analizados, donde se pueden observar valores dispersos y grandes variaciones entre la mayoría de los jugadores. Los bases tienen mayor percepción de la carga que el resto de las posiciones, mientras que en los exteriores e interiores se muestran valores similares, sin embargo, en todas las posiciones hay jugadores concretos que muestran valores distintos respecto al resto de jugadores de su misma posición, esta diferenciación sucede en todos los valores de carga analizados.

\begin{tabular}{|c|c|c|c|c|}
\hline Posición & Carga Media & Înd. Monotonía & Carga Total & Înd.Fatiga \\
\hline Base 1 & $632.59 \pm 538.24$ & $1.49 \pm 1.50$ & $3651.40 \pm 1630.76$ & $6091.06 \pm 5900.95$ \\
\hline Base 2 & $464.99 \pm 195.82$ & $1.11 \pm 0.55$ & $3144.10 \pm 1436.55$ & $4104.38 \pm 3138.79$ \\
\hline Bases & $548.79 \pm 118.52$ & $1.30 \pm 0.27$ & $3397.75 \pm 358.72$ & $5097.72 \pm 1404.80$ \\
\hline Exterior 1 & $482.85 \pm 180.44$ & $1.16 \pm 0.44$ & $3379.98 \pm 1263.06$ & $4338.60 \pm 2873.01$ \\
\hline Exterior 2 & $454.08 \pm 235.60$ & $1.15 \pm 0.39$ & $3158.43 \pm 1640.84$ & $3802.79 \pm 3373.25$ \\
\hline Exterior 3 & $569.00 \pm 213.09$ & $1.27 \pm 0.52$ & $3956.71 \pm 1498.61$ & $5676.48 \pm 3714.97$ \\
\hline Exterior 4 & $536.76 \pm 234.50$ & $1.25 \pm 0.56$ & $3727.62 \pm 1635.65$ & $5448.13 \pm 3825.61$ \\
\hline Exteriores & $510.67 \pm 51.83$ & $1.21 \pm 0.60$ & $3555.68 \pm 355.47$ & $4816.50 \pm 893.41$ \\
\hline Interior 1 & $592.15 \pm 209.03$ & $1.30 \pm 0.50$ & $4115.07 \pm 1463.95$ & $5993.20 \pm 3804.94$ \\
\hline Interior 2 & $490.03 \pm 173.69$ & $1.07 \pm 0.32$ & $3430.19 \pm 1215.84$ & $3917.94 \pm 2183.78$ \\
\hline Interior 3 & $451.62 \pm 278.10$ & $1.16 \pm 0.52$ & $3132.10 \pm 1936.38$ & $4312.07 \pm 3771.25$ \\
\hline Interior 4 & $549.83 \pm 323.09$ & $1.19 \pm 0.60$ & $3822.55 \pm 2264.64$ & $5715.46 \pm 4495.35$ \\
\hline Interiores & $520.91 \pm 62.36$ & $1.18 \pm 0.95$ & $3624.98 \pm 432.09$ & $4984.67 \pm 1023.31$ \\
\hline
\end{tabular}


La Tabla 2 es un resumen de la comparación de variables por posiciones entre bases, exteriores e interiores. Respecto a la variable de carga media, se mostró una magnitud del efecto grande entre los bases y los exteriores. En cuanto al índice de monotonía y el valor de carga total fueron los bases e interiores los que mostraron una magnitud del efecto grande entre ellos. Por último, el índice de fatiga muestra una magnitud del efecto grande entre bases y exteriores. Respecto a la diferencia de medias, los bases y exteriores mostraron las mayores diferencias en las variables de carga media e índice de fatiga, mientras que en las variables de índice de monotonía y carga totales fueron los bases e interiores los que mostraron mayor diferencia de medias.

\begin{tabular}{|c|c|c|c|c|c|}
\hline & & & & & \\
\hline Variable & Posición & & Diferencia & Media & Tamaño \\
\hline Carga Media & Bases & Exteriores & 38.12 & 975.91 & 1.22 \\
\hline & & Interiores & 27.88 & & 0.89 \\
\hline & Exteriores & Bases & -38.12 & & 1.22 \\
\hline & & Interiores & -10.23 & & 0.33 \\
\hline & Interiores & Bases & -27.88 & & 0.89 \\
\hline & & Exteriores & 10.23 & & 0.33 \\
\hline İnd.Monotonía & Bases & Exteriores & 0.09 & 138.46 & 0.9 \\
\hline & & Interiores & 0.12 & & 1.2 \\
\hline & Exteriores & Bases & -0.09 & & 0.9 \\
\hline & & Interiores & 0.03 & & 0.3 \\
\hline & Interiores & Bases & -0.12 & & 1.2 \\
\hline & & Exteriores & -0.03 & & 0.3 \\
\hline Carga Total & Bases & Exteriores & -157.93 & 34471.23 & 0.85 \\
\hline & & Interiores & -227.23 & & 1.22 \\
\hline & Exteriores & Bases & 157.93 & & 0.85 \\
\hline & & Interiores & -69.29 & & 0.37 \\
\hline & Interiores & Bases & 227.23 & & 1.22 \\
\hline & & Exteriores & 69.29 & & 0.37 \\
\hline Índ. Fatiga & Bases & Exteriores & 281.22 & 59369.1 & 1.15 \\
\hline & & Interiores & 113.05 & & 0.46 \\
\hline & Exteriores & Bases & -281.22 & & 1.15 \\
\hline & & Interiores & -168.17 & & 0.69 \\
\hline & Interiores & Bases & -113.05 & & 0.46 \\
\hline & & Exteriores & 168.17 & & 0.69 \\
\hline
\end{tabular}

En la Figura 1 de la progresión de la carga se puede observar como el pico de máxima carga de entrenamiento se mostró en la cuarta semana de competición, mientras que en la semana número 19 se mostró el valor más bajo de carga semanal teniendo en cuenta la media de toda la plantilla.

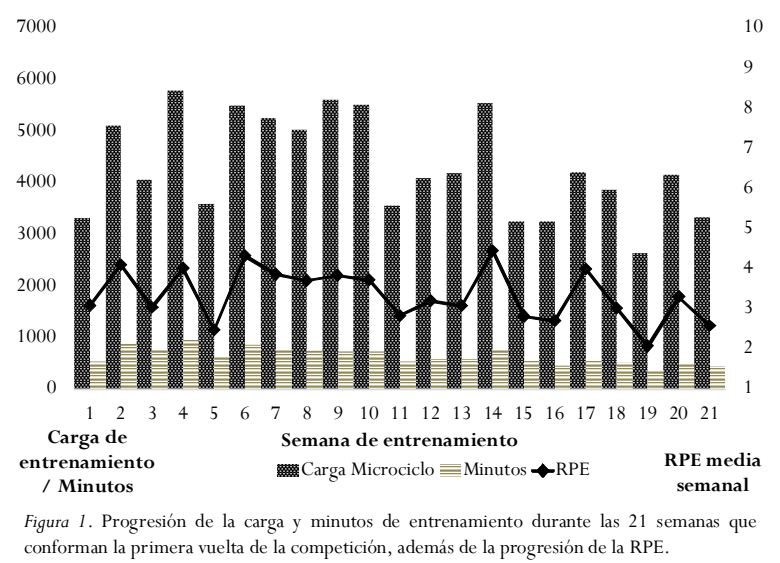

En la Figura 2 se puede observar que la mayoría de los jugadores se mantuvieron en la zona de sweet point perteneciente a valores de ratio de carga aguda-crónica entre 0.7 y 1.3 durante toda la primera vuelta. Referente a la posición de los jugadores, en ninguna se mostraron valores por encima de 1.5 correspondientes a la danger zone. Únicamente los jugadores interiores mostraron valores de ratio por encima de 1.3 las dos primeras semanas de entrenamiento. No se mostraron valores por debajo de 0.7 hasta la semana 11 de temporada, donde los bases muestran por primera vez estos valores, obteniendo también valores inferiores al sweet point en esta posición en las semanas 15 y 19. Los exteriores fueron los que mostraron los valores más regulares en esta variable, siendo la semana 19 la única en la que se obtuvieron valores por debajo del sweet point. Respecto a los interiores, expusieron valores inferiores a 0.7 en cinco semanas de esta primera vuelta, las cuales fueron la 12, 15, 16, 19 y 20. En la semana 19 todos los grupos de posiciones del equipo mostraron valores por debajo de 0.7 de ratio carga aguda-crónica. Fue la única semana de entrenamientos donde todo el grupo de trabajo se mostró fuera del sweet point.

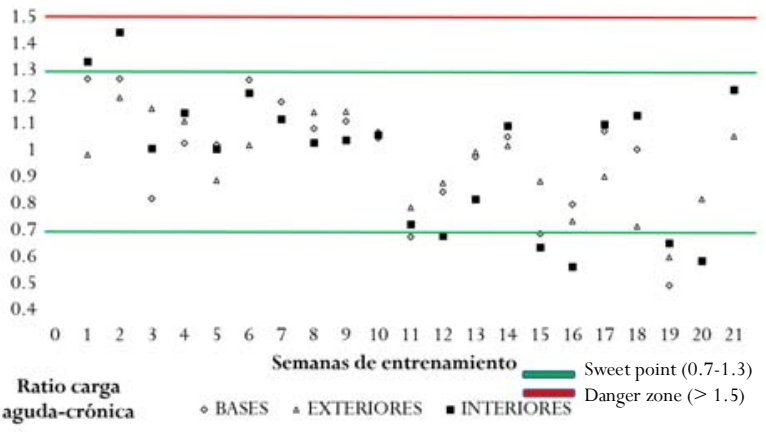

Figura 2. Franjas establecidas para controlar el estado de forma de los jugadores de la plantilla diferenciando sus posiciones respecto a la ratio de carga aguda-crónica, donde el deportista se encuentra en un estado de forma óptimo cuando la ratio está entre 0.7 y 1.3 , mientras que una ratio por encima de 1.5 significaría un estado de fatiga (Gabbett, 2016).

\section{Discusión}

Uno de los objetivos específicos de este estudio fue analizar las diferencias entre las tres posiciones principales que componen un equipo de baloncesto profesional de la categoría LEB Oro, las cuales son bases, exteriores (incluidos escoltas y aleros) e interiores (incluidos ala-pívots y pívots), llevándose a cabo un análisis de diferentes variables tales como: la carga media, semanal y carga total de entrenamiento, índice de monotonía y fatiga y ratio de carga aguda-crónica.

Por un lado, haciendo referencia a los resultados observar cómo los bases tienen una mayor percepción obtenidos según la posición de los jugadores, se puede 
de la carga de entrenamiento, como es el caso del jugador «Base 1», que muestra valores de carga media e índice de fatiga por encima del resto de jugadores. Esto podría deberse a que se trata del base principal del equipo, es decir, el base titular, el cual ha estado sometido a una alta carga de minutos de juego durante estas primeras 21 semanas correspondientes a la primera vuelta de la competición. Respecto a la carga interna, estos resultados son apoyados por el estudio de Fernández-Río (2000) sobre la medición de frecuencias cardíacas medias en jugadores de la segunda división española de baloncesto, que determinó que los bases tenían un promedio de 186 pulsaciones por minuto por partido, mientras que los exteriores tenían 179 pulsaciones por minuto, y los interiores 163 pulsaciones por minuto. Por lo tanto, queda justificado que la posición de base es la que muestra mayores demandas fisiológicas en este aspecto durante un encuentro de baloncesto. Además, desde un punto de vista práctico sería importante tener en cuenta que el estudio de Álvarez (2001) mostró unos resultados donde se analizaban los segundos por minuto que los jugadores permanecían realizando cada actividad con el balón en sus manos y diferenciando entre posiciones, donde los bases tenían el balón 4 segundos por minuto en estas circunstancias, mientras que los exteriores e interiores obtuvieron valores por debajo de un segundo en ambas posiciones. Los resultados del presente estudio también fueron apoyados por la investigación de López y Patricio (2013) sobre los procesos metodológicos de acuerdo a las posiciones de los jugadores y su incidencia en el juego, donde se expone cómo los bases tienen una de las labores de mayor importancia en el equipo, lo que supone un conjunto de esfuerzos físicos, mentales, tácticos, ofensivos y defensivos, lo que podría explicar que los bases, y en este caso, el titular en concreto, haya obtenido valores mucho mayores de carga media e índice de fatiga que el resto del equipo. Sucede algo similar con los jugadores que han disputado mayor carga de minutos durante la competición.

De otra manera, estos resultados son diferentes a los obtenidos en diferentes estudios. Por ejemplo, Puente et al. (2017) determinaron que los jugadores interiores o pívots eran los que menores demandas fisiológicas presentaban durante un partido de baloncesto por su rol y características físicas, mientras que los jugadores de las posiciones bases y aleros, tenían una gran similitud de la percepción de la carga. En contraste, los resultados del presente estudio mostraron ciertas diferencias dado que, en este caso, fueron los exteriores e interiores los que mostraron grandes similitudes de per- cepción de la carga justificadas por magnitudes del efecto pequeñas y moderadas al comparar entre ellos las diferentes variables de la carga, mientras que los bases fueron el caso más aislado, siendo los jugadores con mayor percepción de carga mostrando una magnitud del efecto grande respecto a los interiores y exteriores. Una de las posibles razones de la obtención de diferentes resultados podría deberse al nivel de los jugadores porque la muestra del presente estudio estuvo formada por jugadores de élite con mayor nivel de entrenamiento que el estudio mostrado. Además, dicho estudio (Puente et al., 2017) analizó únicamente un partido de baloncesto, mientras que, el actual estudio estuvo compuesto por 21 semanas de temporada oficial incluyendo entrenamientos y partidos oficiales. Futuras investigaciones realizadas en periodos de entrenamiento más largos son necesarias para contrastar estos resultados.

Haciendo referencia al volumen, algunos estudios han analizado el recorrido aproximado diferenciando las posiciones de bases, exteriores e interiores en el baloncesto (Colli \& Faina, 1987). Estos autores determinaron que los bases recorren un promedio de $3.5 \mathrm{~km}$ por partido, de los cuales aproximadamente $1.1 \mathrm{~km}$ eran a ritmo lento y $1.2 \mathrm{~km}$ a ritmo rápido, los exteriores realizaban un recorrido aproximado de $4.1 \mathrm{~km}$ por partido, de los cuales $1.3 \mathrm{~km}$ eran a ritmo lento, $1.8 \mathrm{~km}$ a ritmo medio y $1 \mathrm{~km}$ a ritmo rápido, y finalmente los interiores, recorrían un promedio de $2.8 \mathrm{~km}$ por partido, siendo $350 \mathrm{~m}$ a ritmo lento, $1.7 \mathrm{~km}$ a ritmo medio y $700 \mathrm{~m}$ a ritmo rápido. Además, otro estudio llevado a cabo en el que se analizaba la media de saltos en cada posición (Hernández-Moreno, 1998) determinó que los bases realizan aproximadamente 27 saltos por partido, los exteriores oscilaron entre 32 y 71, y finalmente los interiores, oscilaron entre 32 y 100 saltos por partido. Observando los promedios de las distancias analizadas y saltos por partido en dichos estudios puede comprenderse la diferente percepción de la carga entre distintas posiciones ante este tipo de carga externa. Sin embargo, los diferentes resultados obtenidos en comparación con este estudio podrían deberse en primer lugar a que se tratan de estudios muy antiguos (1987 y 1998), y el baloncesto ha ido experimentando gran cantidad de cambios respecto a los roles de los jugadores y sus demandas físicas, esto tiene que ver con los cambios de tendencia táctica y la filosofía del juego con el paso del tiempo. Además, el aspecto citado anteriormente sobre los segundos por minuto que los jugadores permanecían realizando cada actividad con el balón en sus manos se podría considerar que es un aspecto en balon- 
cesto que genera mayor percepción de la carga en comparación con el promedio de saltos o el promedio de distancia recorrida (Álvarez, 2001). Por este motivo, aunque los bases muestren menores demandas fisiológicas en algunos aspectos del juego, podrían tener mayor percepción de la carga por aspectos concretos como una mayor posesión del balón.

Cabe destacar que también existen estudios (Montagner et al., 2016) donde se refleja que los jugadores profesionales sufren cierta pérdida de sus capacidades a medida que avanza su carrera deportiva y aumenta la edad. Atendiendo a dicho estudio, se podría concluir que la edad de los jugadores que componen la muestra analizada ha influido en la percepción de la carga, ya que varios jugadores presentaban edades por encima de 35 años.

Focalizando la atención en la progresión de la carga y los minutos, en la Figura 1 se puede observar la progresión de la carga de entrenamiento a lo largo de las semanas y cómo influyen los minutos de juego disputados en los valores de RPE media obtenidos. De manera que, a mayor número de minutos, se han obtenido mayores valores de carga de entrenamiento y RPE media por parte de los jugadores como consecuencia de que se trata de una fórmula donde se multiplica un valor por otro (unidades RPE $\times$ minutos). Schelling (2012) en su estudio sobre la exigencia en el baloncesto relacionado con la carga interna y externa, muestra como cuantos más minutos se encuentra en pista un jugador, más veces experimentará distintas velocidades de desplazamiento tales como sprints, carreras rápidas y moderadas, y trote entre otros, lo que supondrá mayores demandas físicas por los esfuerzos realizados, y por ello, mayor percepción de la carga. Por lo tanto, en jugadores profesionales con experiencia y que han utilizado previamente la RPE, este método podría ser adecuado para realizar este proceso de cuantificación de la carga de entrenamiento.

En cuanto al análisis de la ratio de carga aguda-crónica expuesto en la Figura 2 se han mostrado los valores diferenciando las posiciones de forma que pueden verse aquellos que están en sweet point o valor óptimo de carga (entre 0.7 y 1.3) y aquellos que están fuera de este o por encima de 1.5, lo que supondría un estado de fatiga. De forma que durante esta primera vuelta de competición compuesta por 21 semanas de entrenamiento no se han registrado valores por encima de 1.5 lo que significa que la carga ha sido controlada de forma adecuada. Sin embargo, en algunas semanas los valores se han mostrado fuera del sweet point, obteniendo valores por debajo de 0.7 o por encima de 1.3 . Los únicos valores por encima de 1.3 se han mostrado únicamente en las dos primeras semanas, y ha sido exclusivamente en los jugadores interiores donde se han encontrado estos resultados. Este aspecto se podría entender analizando el estudio de Banister et al. (1975) que muestra que al ser los primeros periodos en los que se comienza a entrenar y donde los jugadores previamente no han estado sometidos a estas cargas de trabajo, el grupo de entrenamiento no se ha ejercitado previamente a esas semanas, y cómo esta ratio de carga aguda-crónica analiza la última semana respecto a las cuatro semanas anteriores, aparecen altos valores al inicio de la temporada. Otro de los estudios recientemente realizados sobre el análisis de la RPE de Ritchie et al. (2016) muestra cómo influye la variación de la RPE en función del momento concreto de temporada, donde especifica que durante la pretemporada se obtuvieron valores más altos de RPE que durante el periodo de competición. Por lo tanto, atendiendo a dicho estudio, cobra sentido que los jugadores interiores muestren altos valores de ratio carga aguda-crónica por encima de 1.3 únicamente las dos primeras semanas de temporada debido a que éstas $\mathrm{CO}-$ rresponden todavía a la pretemporada y no existe competición oficial. Diferentes investigaciones como la de Calvo (1998) muestran una planificación deportiva de una temporada regular donde, durante la pretemporada los jugadores están expuestos a un mayor nivel de carga de entrenamiento. También se obtuvieron valores por debajo de 0.7 , que se podrían explicar por varias razones en comparación con otros estudios. Una de ellas, es por el principio del entrenamiento de supercompensación (Gómez, 2015) en el cual se expone que los jugadores necesitan una adaptación a la carga, es decir, una vez que estos se han acostumbrado a mantener un umbral de entrenamiento exigente por un tiempo prolongado, se adaptan y soportan mejor las cargas de entrenamiento. Aunque exista una disminución progresiva de la carga objetiva de entrenamiento conforme avanza la temporada, un mismo nivel de carga objetiva de entrenamiento podría traducirse en unos niveles menores de carga subjetiva por parte de los jugadores debido a su adaptación y acondicionamiento físico. Finalmente, otro principio del entrenamiento que podría explicar dichos resultados sería el de regeneración periódica debido a que ciertas semanas han tenido un objetivo de descarga.

\section{Limitaciones, aplicaciones prácticas y futu- ras líneas de investigación}


Cabe destacar algunas fortalezas y limitaciones del estudio. El tamaño muestral fue una de las principales fortalezas del estudio porque se ha podido realizar el análisis y control de 10 jugadores, tratándose de una muestra representativa de la categoría teniendo en cuenta el total de jugadores que componen la categoría LEB Oro. Además, todos ellos contaban con una familiarización previa de la RPE (2.4 años de antigüedad en el club) y pertenecían a una misma plantilla. En contraste, una de las principales limitaciones del análisis en cuestión podrían haber sido las lesiones que han sufrido ciertos jugadores durante la realización del estudio, lo que ha podido modificar algunos de los resultados obtenidos. Otra limitación característica de los métodos subjetivos podría ser que durante este estudio se han analizado variables teniendo en cuenta aspectos subjetivos por parte de los jugadores que componen la muestra, pudiendo estar condicionadas por muchos factores ajenos al entrenamiento deportivo. Finalmente, como última limitación, se aclara que las cargas de entrenamiento y competición se han medido conjuntamente.

Respecto a las aplicaciones prácticas y futuras líneas de investigación se concluye que el coste económico necesario para llevar a cabo este tipo de estudios mediante estos métodos es mínimo. Esto justifica que mediante estos procedimientos se podrían realizar futuros estudios de las mismas características o similares, además de que sería posible llevar a cabo un control individualizado y grupal de un equipo en concreto, como ha sido en este caso, de toda la plantilla, y con ello determinar su estado de forma y necesidades en función del momento de la temporada. Actualmente, existen numerosos medios y métodos de cuantificación de la carga que permiten planificar y analizar las distintas semanas de entrenamiento, de forma que se reduzca el riesgo de lesión y se pueda sacar el máximo rendimiento posible a los jugadores de un equipo. Desde el punto de vista práctico, este estudio servirá a futuros entrenadores y preparadores físicos para adecuar la exigencia o carga de entrenamiento tanto de los bases como del resto de posiciones. Futuras investigaciones realizadas en periodos de entrenamiento más largos y con un mayor tamaño muestral son necesarias para contrastar estos resultados. En futuros estudios también se podría realizar esta cuantificación de la carga de entrenamiento y competición en diferentes categorías y comprobar las diferencias respecto a jugadores profesionales como los que forman parte de la muestra de este estudio.

\section{Conclusiones}

Estas conclusiones hacen referencia a jugadores de un equipo de baloncesto profesional de la categoría LEB Oro durante 21 semanas de entrenamiento y competición:

Los bases presentaron una mayor percepción de la carga de entrenamiento y competición obtenida mediante el método RPE sesión en comparación con los exteriores e interiores, que percibieron la carga de manera similar.

El análisis de la carga objetiva y subjetiva mediante cuestionarios diarios de escalas de esfuerzo percibido (RPE) y control de minutos totales de entrenamiento y competición podría ser un método adecuado para realizar el proceso de cuantificación de la carga de entrenamiento y competición.

Respecto a la ratio de carga aguda-crónica, la mayoría de los jugadores de la plantilla se encontraron en el sweet point durante la primera vuelta de la competición. Únicamente los jugadores interiores mostraron valores de ratio carga aguda-crónica por encima de 1.3 durante las dos primeras semanas de entrenamientos en las cuales se acumuló un mayor volumen de entrenamiento. No se mostraron valores de ratio carga agudacrónica por encima de 1.5 en ninguna de las posiciones.

\section{Agradecimientos}

Agradecimiento especial tanto al preparador físico como al entrenador y cuerpo técnico por la colaboración en los datos obtenidos y el trato recibido, así como la predisposición mostrada por parte de los jugadores de la plantilla, pertenecientes al equipo profesional de baloncesto de la categoría LEB Oro, que participaron voluntariamente en este estudio.

\section{Referencias}

Abdelkrim, N. B., El Fazaa, S., \& El Ati, J. (2007). Time-motion analysis and physiological data of elite under-19-year-old basketball players during competition. British journal of sports medicine, 41(2), 69-75.

Alexiou, H., \& Coutts, A. J. (2008). A comparison of methods used for quantifying internal training load in women soccer players. International journal of sports physiology and performance, 3(3), 320-330.

Álvarez, J. C. B. (2001). El análisis de los indicadores externos en los deportes de equipo: baloncesto. Lecturas: Educación Física y Deportes. Revista digital, 38(7), 12.

Arjonilla López, N. (2010). Incidencia de los factores distancia, tiempo, fatiga y concentración de la efectividad en el baloncesto (Tesis doctoral). 
Universidad de Las Palmas de Gran Canaria.

Banister, E. W., Calvert, T. W., Savage, M. V., \& Bach, T. (1975). A systems model of training for athletic performance. Aust J Sports Med, 7(3), 57-61.

Beltrán, A.A. R., Moreno, J. R. R., Siquéiros, M. G. R., Cruz, G. H., \& Fimbres, R. A. G. (2019). Diferencias de carga externa de entrenamiento en ejercicios de baloncesto por medio de acelerometría. Revista de Ciencias del Ejercicio FOD, 14(2).

Bishop, D., Edge, J., Davis, C., Dawson, B., \& Goodman, C. (2002). The importance of buffer capacity (B) for repeated-sprint ability (RSA). In The importance of buffer capacity (B) for repeated-sprint ability (RSA) (p. 101). Australian Association for Exercise and Sports Science.

Bourdon, P. C., Cardinale, M., Murray, A., Gastin, P., Kellmann, M., Varley, M. C. \& Cable, N.T. (2017). Monitoring athlete training loads: consensus statement. International journal of sports physiology and performance, $12(\mathrm{~s} 2), \mathrm{S} 2-161$.

Calleja-González, J., \&Terrados, N. (2009). Indicadores para evaluar el impacto de carga en baloncesto. Revista andaluza de medicina del deporte, 2(2), 56-60.

Calvo, A. L. (1998). Adecuación de la preparación física en el entrenamiento técnico-táctico en baloncesto. Revista Digital (periódico on line), 12.

Campos, M.A., \&Toscano, F. J. (2014). Monitorización de la carga de entrenamiento, la condición física, la fatiga y el rendimiento durante el microciclo competitivo en fútbol. Fútbolpf: Revista de Preparación Física en el Fútbol, 12, 23-36.

Casas, A. (2008). Fisiología y metodología del entrenamiento de resistencia intermitente para deportes acíclicos. Journal of Human Sport \& Exercise, 3(1), 23-53.

Colli, R., \& Faina, M. (1987). Investigación sobre el rendimiento en el basket. Red: revista de entrenamiento deportivo, 1(2), 3-10.

Conte, D., Favero, T. G., Lupo, C., Francioni, F. M., Capranica, L., \& Tessitore,A. (2015). Time-motion analysis of Italian elite women's basketball games: individual and team analyses. The Journal of Strength \& Conditioning Research, 29(1), 144-150.

Daniel, J. F., Montagner, P. C., Padovani, C. R., \& Borin, J. P. (2016). Indicators of technical actions and intensity according to players' positions in official matches of the brazilian basketball league. EBalonmano. com: Revista de Ciencias del Deporte, 12(2), 89-96.

Fernández-Río J, Rodríguez-Alonso N,Terrados N, Fernández-García J, \& Perez N. (2000).Valoración fisiológica en jugadores de básquet. Apunts, 35(132), 11-17.

Foster, C. (1998). Monitoring training in athletes with reference to overtraining syndrome. Medicine \& Science in Sports \& Exercise, 30(7), 1164-1168.

Foster, C., Florhaug, J. A., Franklin, J., Gottschall, L., Hrovatin, L. A., Parker, S., Doleshal, P., \& Dodge, C. (2001). A new approach to monitoring exercise training. The Journal of Strength \& Conditioning Research, 15(1), 109-115.

Gabbett,T. J. (2016). The training - injury prevention paradox: should athletes be training smarter and harder? Br J Sports Med, 50(5), 273-280.

Gómez,A. (2015). Principio de Supercompensación.

González-Badillo, J. J., \& Ribas, J. (2002). Bases de la programación del entrenamiento de fuerza. Barcelona: Inde.

Halson, S. L. (2014). Monitoring training load to understand fatigue in athletes. Sports medicine, 44(2), 139-147.

Hernández, H. M. (1998). Análisis de las estructuras del juego deportivo: fundamentos del deporte. Inde.

Hulin, B. T., Gabbett, T. J., Blanch, P., Chapman, P., Bailey, D., \&
Orchard, J. W. (2014). Spikes in acute workload are associated with increased injury risk in elite cricket fast bowlers. British journal of sports medicine, 48(8), 708-712.

López, L., \& Patricio, L. (2013). Los procesos metodológicos de acuerdo con las posiciones de los jugadores y su incidencia en el entrenamiento deportivo de la selección de baloncesto de los estudiantes del ISTD Guayaquil de la ciudad de Ambato.

Montgomery, P. G., Pyne, D. B., \& Minahan, C. L. (2010). The physical and physiological demands of basketball training and competition. International journal of sports physiology and performance, 5(1), 75-86.

Mujika, I. (2012). Endurance training. Science and Practice.Vitoria-Gasteiz: Iñigo Mujika.

Murray, N. B., Gabbett,T. J., Townshend,A. D., \& Blanch, P. (2017). Calculating acute: chronic workload ratios using exponentially weighted moving averages provides a more sensitive indicator of injury likelihood than rolling averages. British Journal of Sports Medicine, 51(9), 749-754.

Navarro, A. L. (1999). La utilización del conocimiento de los resultados mediante el procedimiento interrogativo para el aprendizaje de una habilidad deportiva en baloncesto. In Investigaciones breves en psicología del deporte (pp. 231-234). Dykinson.

Oliver, J. L. (2009). Is a fatigue index a worthwhile measure of repeated sprint ability? Journal of Science and medicine in Sport, 12(1), 20-23.

Pérez, D. G. (2017). Periodización táctica:planificación de una temporada en baloncesto de élite (Tesis doctoral). Universidad de Cádiz.

Puente, C., Abián-Vicén, J., Areces, F., López, R., \& Del Coso, J. (2017). Physical and physiological demands of experienced male basketball players during a competitive game. The Journal of Strength \&Conditioning Research, 31(4), 956-962.

Reina, M., Mancha-Triguero, D., García-Santos, D., García-Rubio, J., \& Ibáñez, S. J. (2019). Comparación de tres métodos de cuantificación de la carga de entrenamiento en baloncesto. RICYDE. Revista Internacional de Ciencias del Deporte, 15(58), 368-382.

Rhea, M. R. (2004). Determining the magnitude of treatment effects in strength training research through the use of the effect size. The Journal of Strength \& Conditioning Research, 18, 918-920.

Ritchie, D., Hopkins, W. G., Buchheit, M., Cordy, J., \& Bartlett, J. D. (2016). Quantification of training and competition load across a season in an elite Australian football club. International journal of sports physiology and performance, 11(4), 474-479.

Román, M. R., Rubio, J. G., Medina, A.A., \& Godoy, S. J. I. (2020). Comparación de la carga interna y externa en competición oficial de 3 vs. 3 y 5 vs. 5 en baloncesto femenino. Retos: Nuevas Tendencias en Educación Física, Deporte y Recreación, (37), 400-405.

Schelling, X. (2012). Exigencia en baloncesto: carga externa e interna. Deportes, salud y entrenamiento, 11, 6-23.

Uchida, M. C., Teixeira, L. F., Godoi, V. J., Marchetti, P. H., Conte, M., Coutts, A. J., \& Bacurau, R. F. (2014). Does the timing of measurement alter session-RPE in boxers? Journal of sports science \&medicine, 13(1), 59.

Vargas Fuentes, A., Urkiza Ibaibarriaga, I., \& Gil Orozko, S. M. (2015). Effect of preseason matches in sports planning:Variability of training sessions. Retos. Nuevas Tendencias en Educación Física, Deporte y Recreación, (27), 45-51.

Vasconcelos, A. (2005). Planificación y organización del entrenamiento deportivo. Barcelona: Paidotribo.

Williams, S., West, S., Cross, M. J., \& Stokes, K. A. (2017). Better way to determine the acute: chronic workload ratio? British Journal of Sports Medicine, 51(3), 209-210. 